\title{
Research on the Teaching of Copying Translation in Network Environment
}

\author{
Xiaoyan $\mathrm{Ni}^{1 \text {, a }}$ \\ ${ }^{1}$ Jiangxi Police College, Nanchang, Jiangxi, 330103
}

Keywords: Network Environment; Imitation; Translation

\begin{abstract}
With the development of network technology tends to mature, directly to the imitation of translation teaching to bring endless opportunities. But people tend to question the imitation of the network environment imitation, whether the network environment imitation translation teaching evidence to follow if so, how should that go further? What is the effect of imitation translation teaching on existing teaching? In view of the skepticism held by people, this paper studies and discusses these problems, analyzes the support of social learning theory to imitation translation teaching, and discusses the feasibility of imitation translation teaching.
\end{abstract}

\section{Introduction}

Imitation of teaching, in the people's life dictionary of the word appears less, but people's lives are everywhere in the use of examples of imitation teaching examples. In the process of translating and teaching in some teachers, the teaching method is used intentionally or unintentionally. However, at home, there are few specialized studies on imitation translation teaching.

\section{The Basic Theory of Imitation Translation Teaching}

Imitation translation teaching model refers to the search on the network comparable corpus, to find the usual expression, and in the translation to be imitated. According to the study of Bandura's theory of social learning (ie, imitation of learning), human studies are classified as: direct experience learning and learning through demonstration. Most of the human behavior is by observing the society. And observe the results of learning behavior have a direct strengthening, instead of strengthening and self-strengthening function [1].

Therefore, in the process of translation skills training, demonstration observation can effectively enable students to establish translation rules, improve the practical ability of translation. The traditional translation teaching is to provide reference translation, and the reference translation is provided by the students after the translation of the teacher, but in the translation process, the students did not demonstrate precedent can learn and observe, which greatly reduces the student's learning The ability to translate.

But under the big platform of the Internet, students can download massive amounts of web resources, and teachers and students can search for textual text (ie, model text) through network resources. Students in accordance with the model text and then translated, can effectively guide students from the conscious translation of imitation into unconscious independent translation, to promote a virtuous circle of translation learning.

\section{The Network Environment Imitation of the Feasibility of Translation Teaching}

The rapid rise of the Internet to the development of translation teaching and imitation translation teaching has brought new impetus. The feasibility of imitation translation teaching in the network environment is mainly manifested in:

The Internet is a knowledge of the ocean, almost can now say on the Internet, basically meet all the information you need, and make full use of the Internet search can effectively improve the quality of translation. Through the Internet, teachers and students can search a number of model text, and similar text of the Feng Branch, the characteristics of learning, for the future accurate and 
effective translation to lay a good foundation. For example: the introduction of the school, through the search of other schools in English, to understand the characteristics of its style and the article in the same vocabulary in the English and Chinese language corresponding expression, and then imitate it.

Now there are many online dictionaries and online translations online. Excellent online dictionaries can help students reduce unnecessary trouble, and can grasp the new vocabulary in time. Internet translation and so on with real-time translation, vocabulary, terminology update timely, quick search and so on. Improve the efficiency of student translation.

\section{The Network Environment Imitation Translation Teaching Steps}

In the teaching process, teachers can choose the appropriate translation text according to the student level and interest. And according to Ban Dula's theory "to observe the efficiency and level of learning by the demonstration activities of the significance and credibility of the impact," then the teacher in the selection of text should try to choose and students living more contact, and can let Students in the demo to find more comparable text to imitate.

Students can be on the Internet for the original demonstration of the search, as much as possible to find more comparable copies. But in the search process to pay attention to find comparable text must be the same with the original text or similar theme, and comparability is stronger.

In the find comparable text after its study, and proceed to translate, you can effectively imitate. The imitation of the main reflected in:

Language imitation: The purpose of language imitation is to make the translation of the translation of the word professional. I believe that many people will encounter this situation, a word in the dictionary is very understanding of the semantics, but it can not be applied to the sentence, or that is to know the meaning of the word, but often put the word wrong place, Which is caused by improper collocation of the word, and this phenomenon can be collectively referred to as "Chinese-style English". But if there is a comparable text in the case, this situation will be much better.

The original "Xiangtan Electrical and Mechanical College and Hunan Textile College merged to form" for the merger how to express it. For example, it was formed in 2002 through the merger of two previous college.

Hunan Institute of Engineering was created with the merger of Xiangtan was created with the merger of Xiangtan Mechanical and Electrical Engineering College and Hunan Textile College.

It is not difficult to see from this that it is typical of Chinese-style English.

Structural imitation: In the process of translation, student translation is easy to receive the impact of the original structure, which is due to "Chinese-style English." This will appear to be improper translation of students.

In the classroom teaching, may be appropriate for students to group discussions, and when the group's translation has been completed when the group sent representatives to display the translation, the most important thing is to explain the translation process. For example: how to carry out comparable text search, translation process encountered problems and so on. The audience has been actively the corresponding stage of the students and carries out the evaluation of the various groups of the translation. After the discussion of the class, the group should submit the group translation experience. This will enable students to learn the knowledge in the classroom.

There is such a word, no matter how good the actors also need the audience. The teacher should evaluate the translation of each group of students, mainly to encourage the main. This will make the students spend a lot of time in the results of a certain affirmation. But the teacher should also be inadequate on the translation of local spending, and give the appropriate recommendations. And always pay attention to the interaction between students and teachers, or teachers patronize, and students do not have a little reaction, which will appear in the classroom embarrassment, while students will produce aesthetic fatigue. 


\section{The Network Environment Imitation of the Role of Translation Teaching Method}

A large number of networks comparable to the emergence of the text and online dictionaries, online translations, etc. is a huge corpus, students through a word of the study or a translation of the structure of an example, will enhance the initiative between students, play an example The And these are mostly summarized by the students summed up their own things, often the most suitable for their own, easy to be remembered by their own brain, deepen the translation of the impression. This kind of dynamic translation can effectively improve the students' ability of language discrimination [2].

Many times to write similar to the main body of the text, and its analysis and research to help students grasp the title of the text translation style and the characteristics of words for the subsequent translation of the composition played a role in bedding. In the translation of the composition, the text of the text structure, such as imitation, can effectively make the students more smooth and professional translation.

In the translation, many people are blindly to emphasize the originality of translation, but did not learn how to run it. Students turn the translation is gradual, students in the early translation is often helpless, do not know where to start, resulting in retreat of the psychological, fear of rejection and rejection. But if it is imitation of translation, it will greatly reduce the translation of the fear in the students and effectively reduce the psychological burden of students. Students are often the initial stage are afraid of mistakes, and imitation to reduce the error rate, but also increased the students' reading ability and vocabulary ability, thereby increasing the confidence of students.

Do not eat a fat man. Teachers in the choice of the original translation of the time, should pursue the principle of gradual and orderly. All the skills are from simple to complex, according to the level of composition. In the initial translation of the students should choose a less difficult text, which can effectively increase the confidence of students, and then gradually increase in accordance with the student learning situation.

Good memory is not as bad as the pen, a certain intensity of the translation exercises not only allow students to learn the process of knowledge, can effectively consolidate the knowledge has been learned. Multiple copies of the agreed subject or similar topics allow students to be familiar with this approach. This is similar to the "language sense" in English learning.

Imitation is not copied, imitation of the main or to let students learn to learn the method, and copy the translation of the ultimate goal is to hope that students from the imitation translation to independent translation of a change. Imitation translation is just a transition, giving a buffer platform. And imitation translation can effectively improve the ability of students to distinguish language, improve students' sensitivity to comparable text, and thus enable students to high-quality translation until a good self-translation habit.

\section{Conclusion}

Traditional translation teaching is often only focus on translation skills and practice, the general lack of understanding of the statement in the text, and the teacher is only required to be able to understand the line on the line, which is the reason for the low quality of traditional translation. The imitation of the translation is to allow teachers to study the translation and practice to the students, to develop students the ability to self-study, and gradually enhance the ability of students to translate independently.

\section{References}

[1] Tian Lili. The workshop of imitation translation teaching model construction and practice [J]. Anhui literature (the second half), 2016, 10: 133-134 + 143

[2] Tan Fang, Yang Yonghe. The network environment of imitation translation teaching [J]. Chinese translation, 2015, 02: 53-57 